\title{
Early Rebleeding After Stent-Assisted Coiling for Intracranial Vertebral Artery Dissection
}

\author{
Qinghai Huang ${ }^{\mathrm{a}}$, Qiang Li ${ }^{\mathrm{a}}$, Yi Xuc ${ }^{\mathrm{a}}$, Yongwei Zhang ${ }^{\mathrm{a}}$, Jianmin Liu ${ }^{\mathrm{a}, \mathrm{b}}$
}

\begin{abstract}
Endovascular treatment with stent-assisted coiling technique has been an important modality of management of intracranial dissection. Early rebleeding is a fatal complication after endovascular treatment of ruptured aneurysm. We describe a rare case of intracranial vertebral artery dissecting aneurysm with a history of Takayasu syndrome. The patient experienced angiographic confirmed early fatal rebleeding after total occlusion of ruptured aneurysm using combination of stenting and coiling. A covered stent was deployed to control the rebleeding. The management strategy for ruptured intracranial vertebral artery dissection was discused.
\end{abstract}

Keywords: Intracranial aneurysm; Stent; Endovascular treatment; Dissection

\section{Introduction}

Intracranial dissecting vertebral artery aneurysms represent approximately $3 \%$ of all intracranial aneurysms, and subarachnoid hemorrhage from dissecting vertebral artery aneurysms is a well-known phenomenon [1]. In addition, prompt treatment of intracranial dissection in acute phase is utmost importance because of high rate of rebleeding in the unsecured state [2]. Endovascular treatment with stent-assisted coiling technique has been an important modality of management of intracranial dissection [3. 4]. Although early bleed-

Manuscript accepted for publication June 17, 2011

${ }^{a}$ Department of Neurosurgery, Changhai Hospital, Second Military Medical University, Shanghai 200433 China

${ }^{\mathrm{b}}$ Corresponding author: Liu Jianmin, Department of Neurosurgery, Changhai Hospital, Changhai Road 168, Shanghai 200433, China.

Email: chstroke@163.com

doi:10.4021/jnr23w ing is a rare event after endovascular treatment, it's often serious with high mortality and morbidity [5]. We present an angiographic confirmed early rebleeding after stent-assisted coiling in a rare intracranial vertebral artery (VA) dissection patient with history of Takayasu syndrome.

\section{Case Report}

A 59-year-old woman with 20-year history of Takayasu syndrome presented with sudden headache and vomiting. CT scan on admission showed spontaneous subarachnoid hemorrhage (Fig. A). Neurological examination upon admission revealed neck stiffness and palsy of left oculomotor nerve, which relieved 2 days later. Digital subtraction angiography showed severe stenosis of bilateral carotid arteries and left vertebral artery (Fig. B, C). Injection in right vertebral artery demonstrated a small dissecting aneurysm in V4 segment and collateral circulation to bilateral hemispheres(Fig. D).

The procedure was performed under general anesthesia. A 5F guiding catheter (Envoy, Endovascular Corporation, Miami, FL) was positioned in distal right VA. We firstly tried to basket and fill the aneurysm with Orbit $3 / 4$, however, the attempts to coil alone failed. So we switched to stent-assisted coiling technique. Medication with load dose of aspirin and clopidogrel was administered by rectally at 2 hours before stenting. A Vasco plus 25 microcatheter was navigated distal to the aneurysm over a microwire $(0.014 \mathrm{inch})$. The delivery system of LEO stent ( $4.5 \mathrm{~mm}$ in diameter, $25 \mathrm{~mm}$ in length; Balt, Montamerency, France) was gently advanced into the Vasco microcatheter. When the precisely targeted location was confirmed, the stent was deployed by depressing the pusher. After successful deployment of LEO stent, a Prowler Select microcatheter was introduced into aneurysmal sac. And the aneurysm was totally occluded using detachable coils (Fig. E). The patient experienced no new cerebrovascular symptoms. After the procedure, low molecular weight heparin was routinely subcutaneously injected and dual antiplatelet drugs (75 mg clopidogrel and $300 \mathrm{mg}$ aspirin, daily) were given.

On day 3 after endovascular treatment, the patient complained of sudden severe headache and subsequently lost 

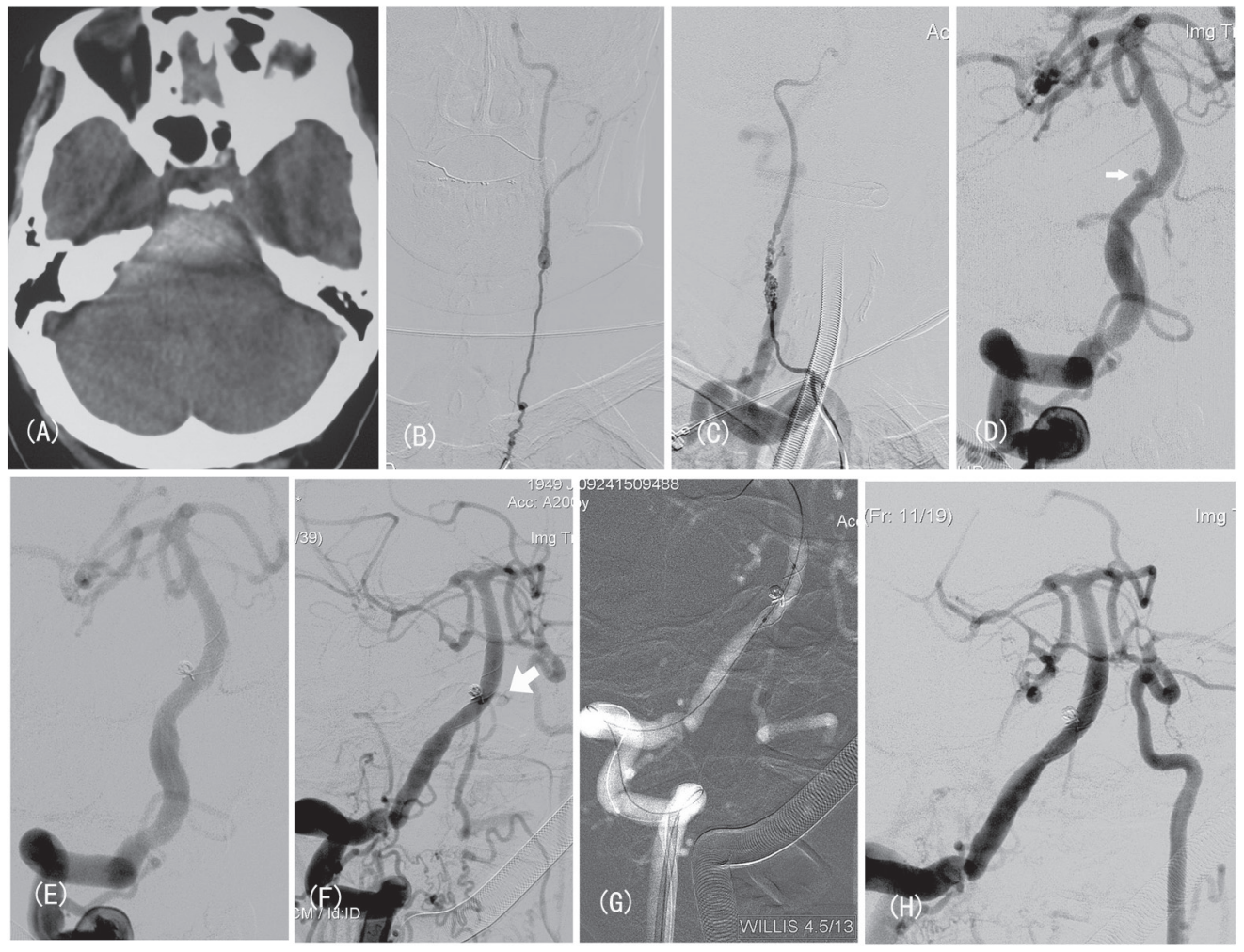

Figure 1. (A) Subarachnoid hemorrhage and hemotoma; (B, C) Bilateral ICA angiogram showed severe stenosis; (D) Injection from R-VA showed a small aneurysm (narrow arrow). (E) Angiogram after stent-assisted coiling demonstrated contrast filling defect and mal-apposition at distal stent edge. (F) Follow-up angiogram confirmed rebleeding (wide arrow). (G, H) The rebleeding controlled by deployment of Willis stent.

consciousness. CT scan demonstrated early rebleeding, which was confirmed by DSA (Fig. F). So we decided to secure the parent vessel using vascular reconstructive technique with covered stent. A 5F ENVOY guiding catheter was introduced into vertebral artery. A novel PTFE-covered stent $(4.5 \mathrm{~mm} / 13 \mathrm{~mm}$,Willis, Microport, Shanghai, China) was advanced over Transend microwire (0.014-inch). After confirmation on the location of stent, we deployed the stent to cover the ruptured site (Fig. G). The control angiogram after stenting showed no bleeding with patency of parent vessel, and poor cerebral perfusion because of intracranial hypertension (Fig. H). Nevertheless, the patient died even in case of extra-ventricular drainage after the procedure.

\section{Discussion}

Rebleeding of ruptured dissecting aneurysms of the vertebral artery has been previously estimated to be between $24 \%$ and $71 \%$ [6-8]. This high rebleeding rate is probably due to the very thin, friable wall of the aneurysm and to a very slow healing process of the vessel. Moreover, most of these rebleeding occurred in acute phase. The poor natural history highlights the importance of emergency management for ruptured VBA dissecting aneurysm.
The treatment of dissecting aneurysm of vertebral artery presenting with hypoplasia of the controlateral VA remains controversial. Sacrifice of the parent artery from which the aneurysm arises, using coil embolization or balloon occlusion, has become a widely accepted approach to treat posterior circulation fusiform aneurysms [9]. However, right vertebral artery in this case is the vessel supplying the majority of brain. Occlusion of this vessel will inevitably result in hypoperfusion of bilateral hemispheres. Recently, more and more reports show the successful use of stent-supported coil embolization in the treatment of fusiform and wide-neck aneurysm. Intraluminal stent deployment can prevent protrusion of coils and change homodynamic in aneurysm sac and parent vessels. As shown by our case, although attempt to coil the aneurysm alone failed, the aneurysm was totally occluded with stent-assisted coiling technique. Some investigators have reported their successful experience on stenting alone or stent-within-a-stent for unruptured dissecting aneurysms [3]. However, the immediate aneurysm obliteration is often not accomplished and the risk of rebleeding can not be totally eliminated. Considering that, reconstructive technique using high porosity of currently available stents alone are not secure for ruptured.

Endovascular treatment using covered stent or stent graft for intracranial vascular diseases were reported, the use of 
these stents for vertebral arteries was limited [10]. The major concern of stenting with covered stent is the occlusion of branch vessels or perforator arteries, since any occlusion of these vessels may result in severe neurological defect [11]. As illustrated by case presented here, the perforator arteries supplying brain stem and anterior spinal artery originate from V4 segment. In addition, stent porosity or the ratio of metal to tissue is another factor associated with neointimal growth. For fear of acute or subacute thrombosis, covered stent or stent graft was not our first choice for our patient. However, in case of early rebleeding, immediate control could be achieved by deployment of stent graft when preservation of patency of parent vessels is necessitated.

Although the high rebleeding rate for intracranial VA dissection in acute phase is well known, the early rebleeding after endovascular treatment is not well-described. Anxionnat et al [12] reported no rebleeding occurred in 17 patients with hemorrhagic intracranial dissections who underwent endovascular treatment and in 2 patients treated surgically. Takagi [2] described in a retrospective review of 62 patients with subarachnoid hemorrhage from vertebral artery dissecting aneurysm, high risk of rebleeding can be predicted by angiographic configurations such as "stenosis and dilation" and "lateral protrusion" and by location at or proximal to the PICA origin. Moreover, the necessity of dual antiplatelet therapy and anticoagulation after stent-assisted coiling or stenting alone increases the risk of intracranial hemorrhage and possibly rebleeding from a ruptured aneurysm.

To our knowledge, this is the first case report of angiographic confirmed early bleeding after stent-assisted coiling for ruptured intracranial vertebral artery dissection.

\section{Acknowledgements}

This work was supported in part by Key Project from the Shanghai Committee of Science and Technology (074119506) and Grant of National Natural Science Foundation (30901556).

\section{References}

1. Santos-Franco JA, Zenteno M, Lee A. Dissecting aneurysms of the vertebrobasilar system. A comprehensive review on natural history and treatment options. Neuro- surg Rev. 2008;31(2):131-140; discussion 140.

2. Takagi T, Takayasu M, Suzuki Y, Yoshida J. Prediction of rebleeding from angiographic features in vertebral artery dissecting aneurysms. Neurosurg Rev. 2007;30(1):32-38; discussion 38-39.

3. Mehta B, Burke T, Kole M, Bydon A, Seyfried D, Malik G. Stent-within-a-stent technique for the treatment of dissecting vertebral artery aneurysms. AJNR Am J Neuroradiol. 2003;24(9):1814-1818.

4. Kim BM, Suh SH, Park SI, Shin YS, Chung EC, Lee $\mathrm{MH}, \mathrm{Kim}$ EJ, et al. Management and clinical outcome of acute basilar artery dissection. AJNR Am J Neuroradiol. 2008;29(10):1937-1941.

5. Bendszus M, Hagel C, Maurer M, Schutz A, Vince GH, Monoranu MC, Solymosi L. Fatal recurrent subarachnoid hemorrhage after complete endovascular aneurysm occlusion. AJNR Am J Neuroradiol. 2006;27(10):20582060.

6. Ramgren B, Cronqvist M, Romner B, Brandt L, Holtas $\mathrm{S}$, Larsson EM. Vertebrobasilar dissection with subarachnoid hemorrhage: a retrospective study of 29 patients. Neuroradiology. 2005;47(2):97-104.

7. Aoki N, Sakai T. Rebleeding from intracranial dissecting aneurysm in the vertebral artery. Stroke. 1990;21(11):1628-1631.

8. Mizutani T, Aruga T, Kirino T, Miki Y, Saito I, Tsuchida T. Recurrent subarachnoid hemorrhage from untreated ruptured vertebrobasilar dissecting aneurysms. Neurosurgery. 1995;36(5):905-911; discussion 912-903.

9. Leibowitz R, Do HM, Marcellus ML, Chang SD, Steinberg GK, Marks MP. Parent vessel occlusion for vertebrobasilar fusiform and dissecting aneurysms. AJNR Am J Neuroradiol. 2003;24(5):902-907.

10. Greenberg E, Katz JM, Janardhan V, Riina H, Gobin YP. Treatment of a giant vertebrobasilar artery aneurysm using stent grafts. Case report. J Neurosurg. 2007;107(1):165-168.

11. Li MH, Zhu YQ, Fang C, Wang W, Zhang PL, Cheng YS, Tan HQ, et al. The feasibility and efficacy of treatment with a Willis covered stent in recurrent intracranial aneurysms after coiling. AJNR Am J Neuroradiol. 2008;29(7):1395-1400.

12. Anxionnat R, de Melo Neto JF, Bracard S, Lacour JC, Pinelli C, Civit T, Picard L. Treatment of hemorrhagic intracranial dissections. Neurosurgery. 2003;53(2):289300; discussion 300-281. 